ARTICLE

DOI: $10.1038 / \mathrm{s} 41467-017-02198-5$

\title{
Bioinspired graphene membrane with temperature tunable channels for water gating and molecular separation
}

\author{
Jingchong Liư ${ }^{1}$ Nü Wang ${ }^{1}$, Li-Juan Yu², Amir Karton², Wen Li³,4, Weixia Zhang ${ }^{4}$, Fengyun Guo ${ }^{1,4}$, \\ Lanlan Hou', Qunfeng Cheng (1) ', Lei Jiang ${ }^{1}$, David A. Weitz ${ }^{4,5} \&$ Yong Zhao ${ }^{1,4}$
}

Smart regulation of substance permeability through porous membranes is highly desirable for membrane applications. Inspired by the stomatal closure feature of plant leaves at relatively high temperature, here we report a nano-gating membrane with a negative temperatureresponse coefficient that is capable of tunable water gating and precise small molecule separation. The membrane is composed of poly ( $\mathrm{N}$-isopropylacrylamide) covalently bound to graphene oxide via free-radical polymerization. By virtue of the temperature tunable lamellar spaces of the graphene oxide nanosheets, the water permeance of the membrane could be reversibly regulated with a high gating ratio. Moreover, the space tunability endows the membrane with the capability of gradually separating multiple molecules of different sizes. This nano-gating membrane expands the scope of temperature-responsive membranes and has great potential applications in smart gating systems and molecular separation.

\footnotetext{
${ }^{1}$ Laboratory of Bioinspired Smart Interfacial Science and Technology of the Ministry of Education, Beijing Key Laboratory of Bioinspired Energy Materials and Devices, School of Chemistry, Beihang University, Beijing 100901, China. ${ }^{2}$ School of Molecular Sciences, The University of Western Australia, 35 Stirling Highway Crawley, Perth, WA 6009, Australia. ${ }^{3}$ Laboratory of Polymer Chemistry, Department of Polymer Materials, College of Materials Science and Engineering, Shanghai University, Nanchen Street 333, Shanghai 200444, China. ${ }^{4}$ John A. Paulson School of Engineering and Applied Sciences, Harvard University, Cambridge, MA 02138, USA. ${ }^{5}$ Department of Physics, Harvard University, Cambridge, MA 02138, USA. Jingchong Liu and Nü Wang contributed equally to this work. Correspondence and requests for materials should be addressed to N.W. (email: wangn@buaa.edu.cn) or to Y.Z. (email: zhaoyong@buaa.edu.cn)
} 
$\mathrm{F}$ or a number of drought-enduring vegetations in nature (such as cacti), the stomata on their leaves close at relatively high temperatures, effectively reducing the water loss in order to sustain life $\mathrm{e}^{1,2}$. This biological phenomenon displays a negative temperature-response characteristic from a membrane perspective, that is, the membrane's permeability decreases as the surrounding temperature increases ${ }^{3-5}$. Although this negative temperature-responsive phenomenon is prevalent in nature, most man-made temperature-responsive membranes have the opposite responsive trend where water permeability increases with increasing temperature, i.e., they show a positive temperatureresponse characteristic ${ }^{6-9}$. This is because most temperatureresponsive polymeric materials adopted in gating membranes undergo a coil-globule shrinking transition of their chains at a lower critical solution temperature $(\mathrm{LCST})^{10-12}$, and as a result, the membrane pores switch from a closed state below the LCST to an open state above the LCST.

Graphene oxide (GO) is a two-dimensional material consisting of a carbon lattice and oxygen-containing groups ${ }^{13}$. Recently, GO membranes (GOMs) have exhibited superior gating and separation performances due to their unique unimpeded twodimensional nanochannels and nacre like lamellar structure $^{14,15}$. The ordered brick and mortar assembly of inorganic and/or organic layers in GO-based membranes endows them with strong interfacial interactions and excellent chemical stability ${ }^{16}$. Therefore, GO-based membranes have been successfully applied to gas, molecular, and organic solvent separations ${ }^{17-30}$. Moreover, controlling molecule penetration through GO-based membranes has received considerable attention due to the crucial importance of gatekeepers control the movement of substances, mimicking living organisms ${ }^{31,32}$. Previous studies have demonstrated that water permeance of different kinds of GO-based membranes can be regulated by external fields such as $\mathrm{pH}$, solvent, ion concentration, electric and magnetic fields ${ }^{20,33-35}$. However, these systems rely on specific chemical environments like ionic strength, acid-base reactions and so on, which significantly limit their scope of practical applications. Temperature controlled gating membrane systems, which are convenient and less dependent on the chemical environment, have more extensive adaptability $^{36}$. However, negative temperature-response graphene-based membranes that can achieve water gating and molecular separation have never been reported.

Here, we construct a negative temperature-response nanogating membrane by covalently grafting poly $(\mathrm{N}$-isopropylacrylamide) (PNIPAM) chains on GO sheets. The water permeance of this membrane varies from $12.41 \mathrm{~m}^{-2} \mathrm{~h}^{-1}$ bar $^{-1}$ at $25^{\circ} \mathrm{C}$ to $1.8 \mathrm{l} \mathrm{m}^{-2} \mathrm{~h}^{-1} \mathrm{bar}^{-1}$ at $50^{\circ} \mathrm{C}$ with a high gating ratio of $\sim 7$. Moreover, the tunable lamellar spacing of the GOM enables it to separate small molecules with different sizes by regulating the temperature. This smart membrane shows promise in many fields, such as fluid transport systems, microfluidic chip systems and molecular separation devices.

\section{Results}

Fabrication of the PNIPAM covalently grafted GOMs. The temperature-responsive PNIPAM covalently grafted GO membranes(P-GOMs) are based on regulating the layer spacing through tuning the molecular configuration of PNIPAM. The two-step construction process and water gating property of $\mathrm{P}$ GOMs are illustrated in Fig. 1, involving polymerization of $N$ isopropylacrylamide (NIPAM) on GO sheets to form PNIPAMgrafted $\mathrm{GO}(\mathrm{P}-\mathrm{GO})$ and assembling $\mathrm{P}-\mathrm{GO}$ into P-GOMs through pressure-driven filtration. When the temperature is below the LCST of P-GO, the PNIPAM chains on P-GO exhibit a swollen coil structure because of hydrogen bond interaction between amide group of PNIPAM and water molecules. Under this condition, the space between two adjacent GO sheets provides appropriate nanochannels for water transport. If the temperature increases above its LCST, the PNIPAM chain shrinks because the hydrogen bond interaction between the amide group and water molecule is replaced by the intramolecular/
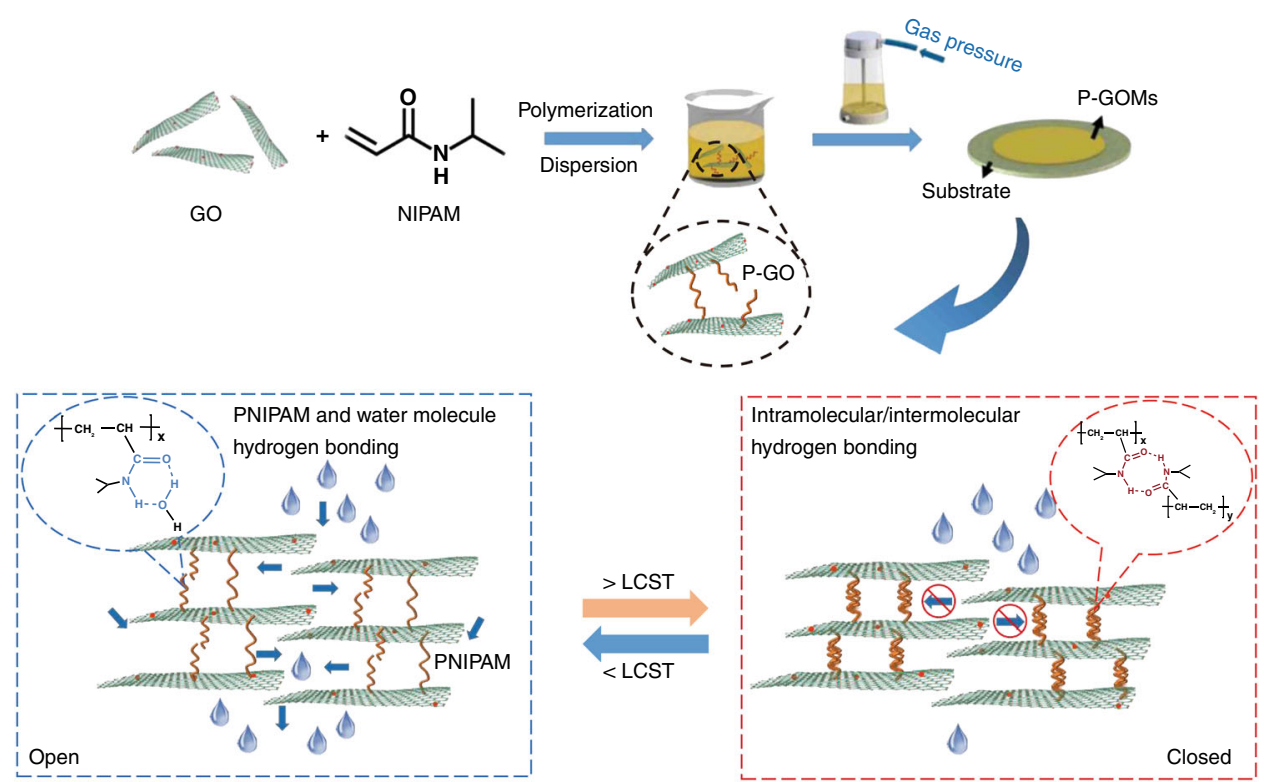

Fig. 1 Fabrication process of the temperature-responsive membrane and its water gating property. $N$-isopropylacrylamide (NIPAM) monomers polymerize on graphene oxide (GO) sheets to form PNIPAM-grafted GO (P-GO). Then P-GO assembles into PNIPAM covalently grafted GO membranes (P-GOMs) through pressure-driven filtration of their aqueous dispersion. The water permeance of $\mathrm{P}-\mathrm{GOMs}$ could be regulated via the environmental temperature ( $T$ ). P-GOMs have a large water permeance when $T$ is below its lower critical solution temperature (LCST) because of the expanded water flow channel caused by the swollen PNIPAM chains. The water permeance decreases when $T$ is above the LCST because of the narrowed channel of P-GOMs, which is caused by the shrink of PNIPAM chains 
a

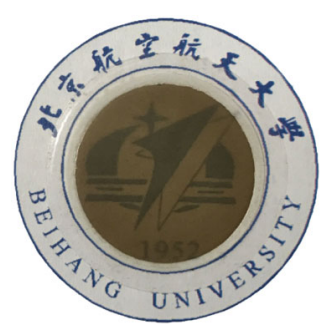

d

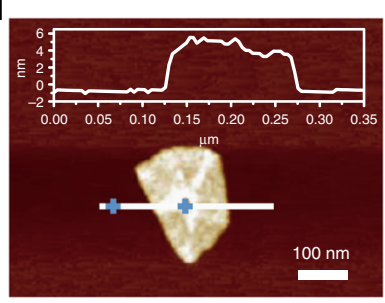

b
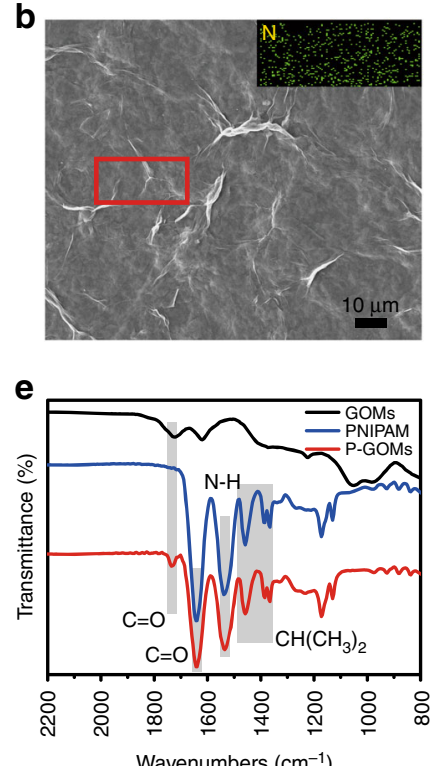
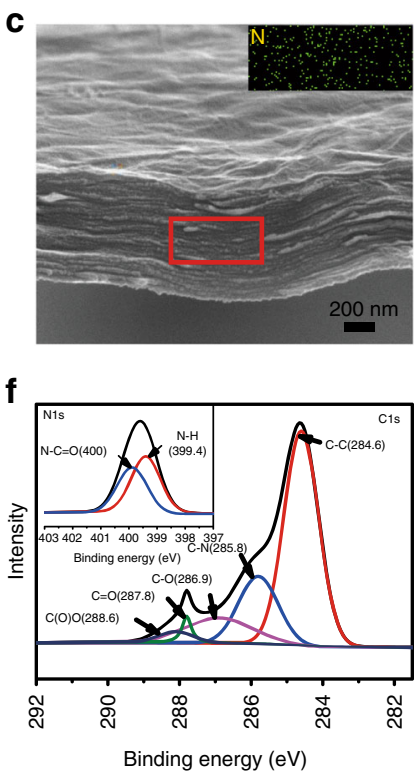

Fig. 2 Characterization of P-GOMs. a Digital photo of P-GOMs. b, c Top-view and cross-sectional SEM images of P-GOMs showing a typical wrinkle surface and stacking microstructure. The insets are EDS maps indicating that nitrogen is evenly distributed on both surface and cross-section of P-GOMs. $\mathbf{d}$ AFM image of a single P-GO sheet with thickness $\sim 5 \mathrm{~nm}$. e ATR-FTIR spectra of GOMs, PNIPAM and P-GOMs, illustrating covalent bound between PNIPAM and GO. $\mathbf{f}$ XPS spectrum of P-GO, of which C1s region fitting into five peaks at 284.6, 285.8, 286.9, 287.8, and 288.6 eV, representing C-C, C-N, $\mathrm{C}-\mathrm{O}, \mathrm{C}=\mathrm{O}$ and $\mathrm{C}(\mathrm{O}) \mathrm{O}$. Inset is N1s region, fitting into two peaks at 399.4 and $400.0 \mathrm{eV}$, representing amine and amide, respectively, because of the graft of PNIPAM

intermolecular hydrogen bonding of PNIPAM ${ }^{37}$. In this process, the GO sheets are pulled closer because they are covalently tethered to the ends of PNIPAM chains, resulting in a smaller lamellar distance of P-GOMs. Then, the water permeance decreases as water is mostly blocked by P-GOMs. It is thereby feasible to construct P-GOMs with negative coefficient in hydraulic permeability.

The temperature-responsive P-GOMs were obtained by pressure-assisted self-assembly technology ${ }^{38}$ with the freezedried P-GO (Supplementary Figs. 1a and b). The P-GOMs displayed typical brown color with good optical transparency and uniform surfaces without defects (Fig. 2a and Supplementary Fig. 1c), which was similar to GOMs (Supplementary Fig. 1d) ${ }^{19}$. The surface and cross-section scanning electron microscope images of the P-GOMs (Fig. 2b, c) demonstrate that the P-GOMs have wrinkled surface and stacking microstructure like normal GOMs (Supplementary Figs. 2a and b). The energy dispersive spectroscopy results show that nitrogen element is evenly distributed in both surface and cross-section of P-GOMs (Fig. 2b, c), whereas almost no nitrogen is detected in pristine GOMs (Supplementary Fig. 2). These are side evidences of the existence of PNIPAM between the GO laminates. After the polymerization of NIPAM on GO sheets, the thickness of a single P-GO sheet increased to $\sim 5 \mathrm{~nm}$ as shown by the atomic force microscope (AFM) image in Fig. 2d. Hence the thickness of the PNIPAM layer was $\sim 4 \mathrm{~nm}$ considering the $0.8 \mathrm{~nm}$ thickness of pristine GO sheet (Supplementary Fig. 3). To further examine the interactions between GO and PNIPAM, attenuated total reflectance Fourier transform infrared (ATR-FTIR) measurements of GOMs, pure PNIPAM, and P-GOMs were conducted (Fig. 2e). Besides the stretching peak of oxygen functional groups of GO at $1743 \mathrm{~cm}^{-1}$, several new peaks at 1642, 1540, and $1367 \sim 1460 \mathrm{~cm}^{-1}$ appeared after grafting with PNIPAM ${ }^{39}$. These new peaks are attributed to the $\mathrm{C}=\mathrm{O}$ stretching (amide I band), the deformation of $\mathrm{N}-\mathrm{H}$ bond and $\mathrm{CH}\left(\mathrm{CH}_{3}\right)_{2}$ groups of PNIPAM, respectively ${ }^{40,41}$. These characteristic peaks coincided with the spectrum of pure
PNIPAM. During the reaction, NIPAM not only polymerized on GO surfaces but also formed free PNIPAM through selfpolymerization $^{42}$. To eliminate the possibility that the new peaks detected in the ATR-FTIR spectrum of P-GOMs were from the free PNIPAM attached to GO, the filtrate of the P-GOMs construction process was also analyzed by ATR-FTIR (Supplementary Fig. 4). There was no absorption peak of PNIPAM in the spectrum of the filtrate, confirming the strong covalent interaction between PNIPAM and GO. The X-ray photoelectron spectroscopy (XPS) spectra of GO and P-GO clearly indicate the introduction of nitrogen-containing functional groups after modifying GO with PNIPAM (Fig. 2f and Supplementary Fig. 5). The C1s XPS spectrum of P-GO shows curve fittings with a new peak of C-N at the binding energy of $285.8 \mathrm{eV}$ compared with that of $\mathrm{GO}^{43}$. More characterization including Raman spectra, and ${ }^{1} \mathrm{H}$ nuclear magnetic resonance also demonstrate that PNIPAM chains have been successfully covalently grafted to GO sheets (Supplementary Figs. 6 and 7).

Temperature-responsive property of the P-GOMs. As is well known, PNIPAM will undergo a phase transition behavior in water and form aggregates (which makes the solution turbid) when the temperature is above its LCST. This reversible temperature-response property of PNIPAM, as expected, was well retained after it was covalently bound to GO. Figure 3 a shows that the P-GO reversibly dispersed and aggregated upon cooling and heating of the aqueous dispersion between $25^{\circ} \mathrm{C}$ and $50^{\circ} \mathrm{C}$. This phenomenon was not observed for the GO aqueous dispersion (Supplementary Fig. 8), indicating that GO does not have any temperature-response property. As reflected by the variabletemperature ultraviolet visible (UV-vis) spectrum, the LCST of P$\mathrm{GO}$ was $\sim 30^{\circ} \mathrm{C}$ (determined by the temperature point where the absorption value dramatically increased), which was slightly lower than that of pure PNIPAM $\left(\sim 32^{\circ} \mathrm{C}\right)^{44}$ (Supplementary Fig. 9). This small variation was because $\mathrm{GO}$ was partially reduced 
a
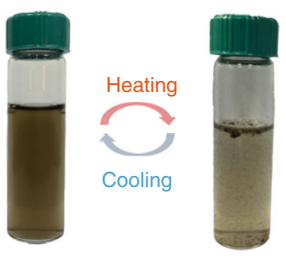

b

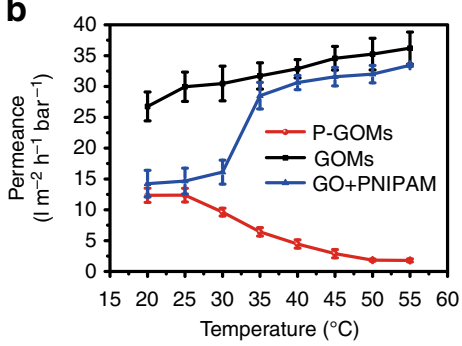

e

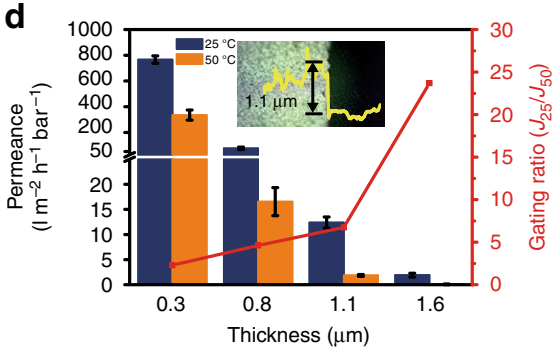

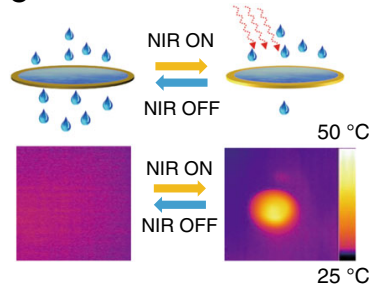

C

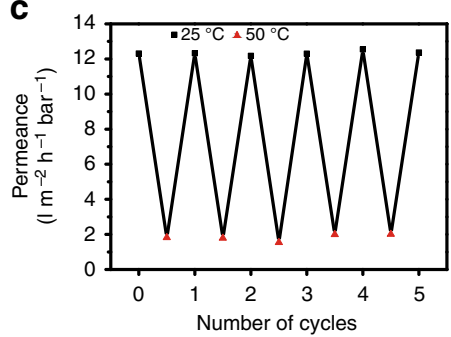

f

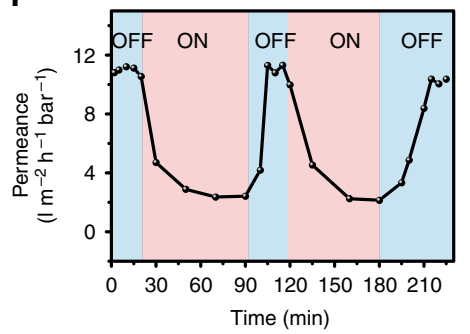

Fig. 3 Thermal- and photo-gating performances of P-GOMs. a P-GO sheets re-dispersed and aggregated upon cooling and heating of the aqueous dispersion between $25^{\circ} \mathrm{C}$ and $50^{\circ} \mathrm{C}$, indicating the temperature-responsive property of P-GO. b Temperature-dependent water permeance curves of GOMs, P-GOMs and physically blending PNIPAM/GO membranes, P-GOMs show the unique negative temperature-responsive coefficient, which is opposite to the positive coefficient of GOMs and GO/PNIPAM blending membrane. Error bars, s.d. $(n=3)$. c Reverse stability test of the temperatureresponsive water gating property of P-GOMs. $\mathbf{d}$ Thickness-dependent water permeance and gating ratio of P-GOMs, $1.1 \mu \mathrm{m}$ thickness is adopted after balancing the water permeance and high gating ratio. Error bars, s.d. $(n=3)$. e Schematic showing the water permeance of P-GOMs decreased after irradiation of near-infrared (NIR). The bottom pictures are thermographic images of P-GOMs before and after irradiation of NIR. $\mathbf{f}$ The water permeance of P-GOMs decreases when switching on NIR light and returns to initial values when the NIR light is off

after functionalization and thus became a little hydrophobic. As a result, the LCST of PNIPAM decreased when bound to hydrophobic groups ${ }^{45}$. This hypothesis was supported by the results of the XRD, water contact angle, XPS, and Raman characterizations (Supplementary Fig. 10).

These results indicate that PNIPAM is successfully grafted onto GO surfaces by covalent bonds, making it feasible to act as water gating by virtue of the temperature tunable lamellar spaces of the P-GOMs. Then we performed pressure-driven hydraulic permeability experiments to study the temperature-response behavior of P-GOMs. As controls, GOMs and physically blended GO/ PNIPAM membrane were also compared. As illustrated in Fig. 3b, the P-GOMs exhibited the negative temperatureresponse characteristic, which was distinctly different with the GOMs and physically blended GO/PNIPAM membrane. The water permeance of GOMs slightly increased as the temperature went up, owing to the decreased water viscosity ${ }^{46}$. For the physically blended GO/PNIPAM membranes, the permeance had a sharp transition when the temperature changed from $30^{\circ} \mathrm{C}$ to $35^{\circ} \mathrm{C}$. Given that the LCST of PNIPAM is $\sim 32^{\circ} \mathrm{C}$, the PNIPAM chains non-covalently attached to GO in the membranes with shrunken conformation expanded the size of the channels at $35^{\circ}$ $\mathrm{C}$ compared with that at $30^{\circ} \mathrm{C}$, thus resulting in a larger permeance increase (Supplementary Fig. 11) ${ }^{36}$. For our P-GOMs, however, the permeability decreased with the temperature increasing when the temperature was above $30^{\circ} \mathrm{C}$, showing a negative temperature-response characteristic. The P-GOMs (1.1 $\mu \mathrm{m}$ thickness) had an average water permeance of $12.41 \mathrm{~m}^{-2} \mathrm{~h}^{-1}$ bar $^{-1}$ at $25^{\circ} \mathrm{C}$, and decreased to $1.81 \mathrm{~m}^{-2} \mathrm{~h}^{-1} \mathrm{bar}^{-1}$ when the temperature increased to $50{ }^{\circ} \mathrm{C}$. The gating ratio of P-GOMs $\left(J_{25} / J_{50}\right.$, where $J_{T}$ is the water permeance at temperature $T^{\circ} \mathrm{C}$ ) was $\sim 7$ and sustained after several cycles (Fig. 3c). Therefore, the P-GOMs can serve as a hydraulic permeability gating membrane with a relatively large negative gating ratio. Furthermore, we studied the thickness effect of P-GOMs on the gating ratio as shown in Fig. $3 \mathrm{~d}$. Assuming that water inside the nanochannels behaves as a viscous flow $^{29,30,47,48}$, the water permeated through the P-GOMs can be described by the Hagen-Poiseuille equation:

$$
J=\varepsilon \pi r^{2} \Delta p / 8 \eta d \tau
$$

where $J$ is the water permeance, $\varepsilon$ is the surface porosity, $r$ is the pore radius, $\Delta p$ is the applied pressure, $\eta$ is the water viscosity, $d$ is the membrane thickness and $\tau$ is the tortuosity. According to Equation (1), the water permeance is inversely proportional to the membrane thickness. The thickness variations of P-GOMs with different loading amounts of P-GO aqueous dispersion can be acquired from Supplementary Fig. 12. The water permeance decreased at both of $25^{\circ} \mathrm{C}$ and $50^{\circ} \mathrm{C}$ as the thickness of P-GOMs increased from 0.3 to $1.6 \mu \mathrm{m}$, whereas the gating ratio increased gradually. Although the gating ratio of 23.7 was able to reach when the thickness of P-GOMs was $1.6 \mu \mathrm{m}$, the low permeance of only $1.91 \mathrm{~m}^{-2} \mathrm{~h}^{-1} \mathrm{bar}^{-1}$ at $25^{\circ} \mathrm{C}$ would restrict its scope of applicability. Thus, the P-GOMs with thickness of $1.1 \mu \mathrm{m}$ were chosen for the temperature-response gating experiments.

Besides directed thermal tunable permeation, we realized remote control of the membrane permeability via light because of the superior light-to-heat conversion capability of GO. This would be implemented more conveniently in deserved applications ${ }^{14,32,49}$. When the P-GOMs were exposed under $808 \mathrm{~nm}$ near-infrared (NIR) light at power density of $0.3 \mathrm{~W} \mathrm{~cm}^{-2}$ in air at an initial temperature of $\sim 25^{\circ} \mathrm{C}$, its temperature rose across the LCST $\left(30^{\circ} \mathrm{C}\right)$ (Fig. 3f). Water has a low absorption at $808 \mathrm{~nm}$ NIR while the P-GOMs have a strong absorption, thus the water permeance of the P-GOMs can be regulated by the NIR light (Fig. 3e) $)^{50,51}$. As shown in Fig. 3f, the water permeance was $\sim 11.2$ $1 \mathrm{~m}^{-2} \mathrm{~h}^{-1}$ bar $^{-1}$ at ambient temperature $\left(\sim 25^{\circ} \mathrm{C}\right)$ without the NIR light. After irradiation, the water permeance of P-GOMs decreased to $\sim 2.81 \mathrm{~m}^{-2} \mathrm{~h}^{-1} \mathrm{bar}^{-1}$. The cycle of light-induced temperature-responsive gating performance can be repeated as the Fig. $3 c$ shows without obvious decay. The photocontrol of 

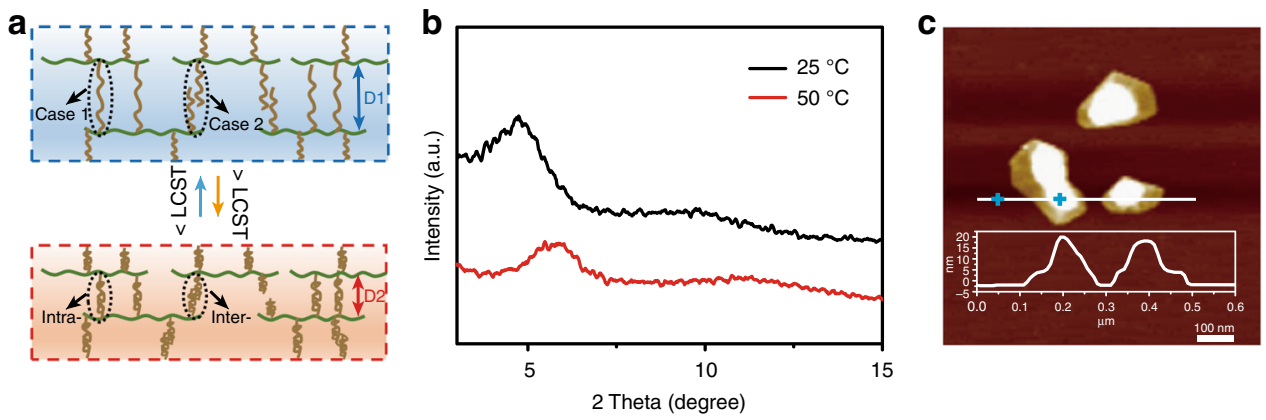

d
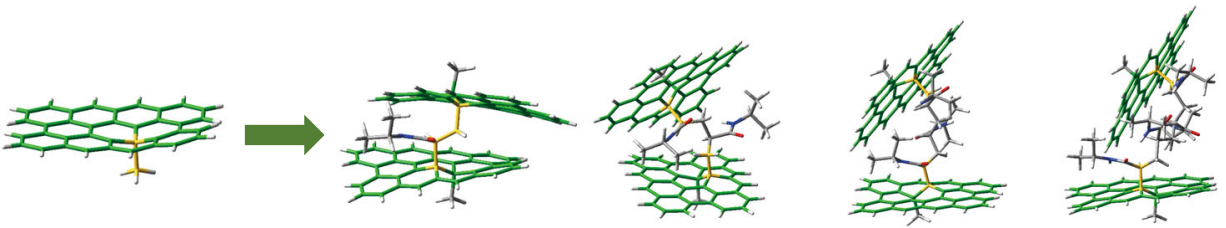

(1b): $-65.2 \mathrm{~kJ} \mathrm{~mol}^{-1}$

(2b): $-154.6 \mathrm{~kJ} \mathrm{~mol}^{-1}$

(3b): $-254.5 \mathrm{~kJ} \mathrm{~mol}^{-1}$

(4b): $-396.0 \mathrm{~kJ} \mathrm{~mol}^{-1}$

Fig. 4 Mechanism of the negative temperature-response behavior of P-GOMs. a Schematic diagram of the channel size change of P-GOMs from D1 to D2 caused by the swelling or shrinking of PNIPAM chains covalent bound to GO sheets. Case 1: PNIPAM chains grow from one GO sheet and terminate at another GO sheet. Intramolecular hydrogen bonding is formed when the temperature is above its LCST. Case 2: PNIPAM chains grow from one GO sheet and terminate freely. Intermolecular hydrogen bonding is formed when the temperature is above its LCST. b The XRD patterns of P-GOMs at $25^{\circ} \mathrm{C}$ and 50 ${ }^{\circ} \mathrm{C}$. The right shift of the diffraction peak indicates the smaller lamellar distance at $50^{\circ} \mathrm{C}$ than $25^{\circ} \mathrm{C}$. c The AFM image of P-GO. The height of crosssectional profile proves that the $\mathrm{P}-\mathrm{GO}$ sheets would like to stack together at $50{ }^{\circ} \mathrm{C}$. d Optimized geometries of $\left[\mathrm{C}_{36} \mathrm{H}_{16}-\mathrm{CH}_{3}\right] \bullet$ and the representative products of reactions (1b) $\mathrm{C}_{36} \mathrm{H}_{16}-\mathrm{CH}_{3}-\mathrm{NIPAM}-\mathrm{CH}_{3}-\mathrm{C}_{36} \mathrm{H}_{16}$, (2b) $\mathrm{C}_{36} \mathrm{H}_{16}-\mathrm{CH}_{3}-(\mathrm{NIPAM})_{2}-\mathrm{CH}_{3}-\mathrm{C}_{36} \mathrm{H}_{16}$, (3b) $\mathrm{C}_{36} \mathrm{H}_{16}-\mathrm{CH}_{3}-\left(\mathrm{NIPAM}_{3}-\mathrm{CH}_{3}-\mathrm{C}_{36} \mathrm{H}_{16}\right.$ and $(4 \mathrm{~b})$ $\mathrm{C}_{36} \mathrm{H}_{16}-\mathrm{CH}_{3}-(\mathrm{NIPAM})_{4}-\mathrm{CH}_{3}-\mathrm{C}_{36} \mathrm{H}_{16}$. Others are shown in Supplementary Fig. 16. The graphene sheet is shown in green tubes, the PNIPAM as tubes (atomic color scheme: $\mathrm{C}$, gray; $\mathrm{N}$, blue; $\mathrm{O}$, red; $\mathrm{H}$, white). The newly formed bond is highlighted in yellow

water transport through P-GOMs may expand their applications such as in lab-on-a-chip settings ${ }^{52}$.

Mechanism of the negative temperature-response behavior of P-GOMs. The unusual negative temperature-response behavior of P-GOMs, which is quite different from the GO/PNIPAM physical blending membranes ${ }^{36}$, is due to the covalently bound interactions between PNIPAM chains and GO sheets (Supplementary Figs. 13 and 14, Supplementary Note 1). When synthesizing P-GO during the free-radical polymerization, there were two cases of the PNIPAM chains termination (except the selfpolymerization of NIPAM): two ends of a PNIPAM chain termination by connecting two GO sheets and terminating freely at the bulk solution with only one end of the chain anchored to GO sheet. The AFM images of $\mathrm{GO}$ and $\mathrm{P}-\mathrm{GO}$ at $25^{\circ} \mathrm{C}$ presented in Supplementary Fig. 15 show that, both single- and multi-layered P-GO sheets exist because of the interactions of PNIPAM chains with GO sheets, whereas almost no stacked sheet is observed for $\mathrm{GO}$ sample. Figure 4a demonstrates that, for the first case, the covalently linked PNIPAM would pull two adjacent GO sheets closer by molecular chain contraction when the temperature is above its LCST. For the second case, part of the PNIPAM chains bound to adjacent GO sheets would form intermolecular hydrogen bonds when the temperature is above its LCST, which narrows the distance between two adjacent GO sheets because of the entanglement of PNIPAM chains ${ }^{3}$. Both these two cases result in a tunable GO lamellar space which play a dominant role in the negative temperature-response gating performance. This hypothesis was proved by XRD and AFM. For the P-GOMs in wet state, because PNIPAM was intercalated into the GO sheets to form a layered nanostructure, the diffraction peak of GO (Supplementary Fig. 10) disappeared and a new peak at $2 \theta=4.71^{\circ}$ appeared. When the temperature increased to $50^{\circ} \mathrm{C}$, the diffraction peak of P-GOMs shifted right to
$2 \theta=5.91^{\circ}$. The smaller lamellar distance at $50^{\circ} \mathrm{C}$ proved the movement of GO sheets because of the shrinking of PNIPAM chains, resulting in a decreased water permeance. Besides, the entanglement of PNIPAM chains between two adjacent GO sheets also results in the decreased water permeance ${ }^{53,54}$. Furthermore, the temperature-response behavior of P-GO was characterized by AFM. The pre-heated P-GO dispersion $\left(\sim 50^{\circ} \mathrm{C}\right)$ was spin-coated $(2000 \mathrm{rpm})$ onto freshly cleaved mica to obtain the AFM sample ${ }^{55}$. As shown in Fig. 4c, the cross-sectional profile indicates the aggregation of P-GO sheets with heights of $\sim 20 \mathrm{~nm}$, implying the dense stacking of $\mathrm{P}-\mathrm{GO}$ sheets at $50^{\circ} \mathrm{C}$.

In order to further understand the mechanism, quantum chemical calculations were performed using density functional theory to elucidate the reaction enthalpy for the formation of the graphene-PNIPAM. In radical polymerization, a polymer forms by the successive addition of radicals. The aim of this computational investigation is to establish whether the reaction of functionalized graphene with NIPAM monomers would terminate composite molecules freely in bulk solution or terminate at another functionalized graphene sheet. The polymerization reaction mechanism for the two possibilities along with the full computational details are given as Supplementary Note 2. Mylvaganam et al. found that the carbon adjacent to the $s p^{3}$ carbon is prone to be attacked by an ethylene moiety ${ }^{56}$. Owing to steric factors the graphene sheet is expected to react with NIPAM molecules on the opposite side of the methyl group. Supplementary Fig. 16 and Fig. 4d show the optimized geometries of these products in reactions $(1 \mathrm{a}-4 \mathrm{a})$ and $(\mathrm{b}-4 \mathrm{~b})$ (Supplementary Note 2). The calculated reaction enthalpies for these two sets of reactions are given in Supplementary Table 1. An important result is that the first two reactions involving one functionalized graphene sheet (1a and 2a) are endothermic, whereas the corresponding reactions including two functionalized graphene sheets $(1 \mathrm{~b}$ and $2 \mathrm{~b})$ are exothermic. Specifically, the reaction enthalpies at $298 \mathrm{~K}\left(\Delta H_{298}\right)$ were $+50.9(1 \mathrm{a}),+20.8(2 \mathrm{a}),-65.2$ 

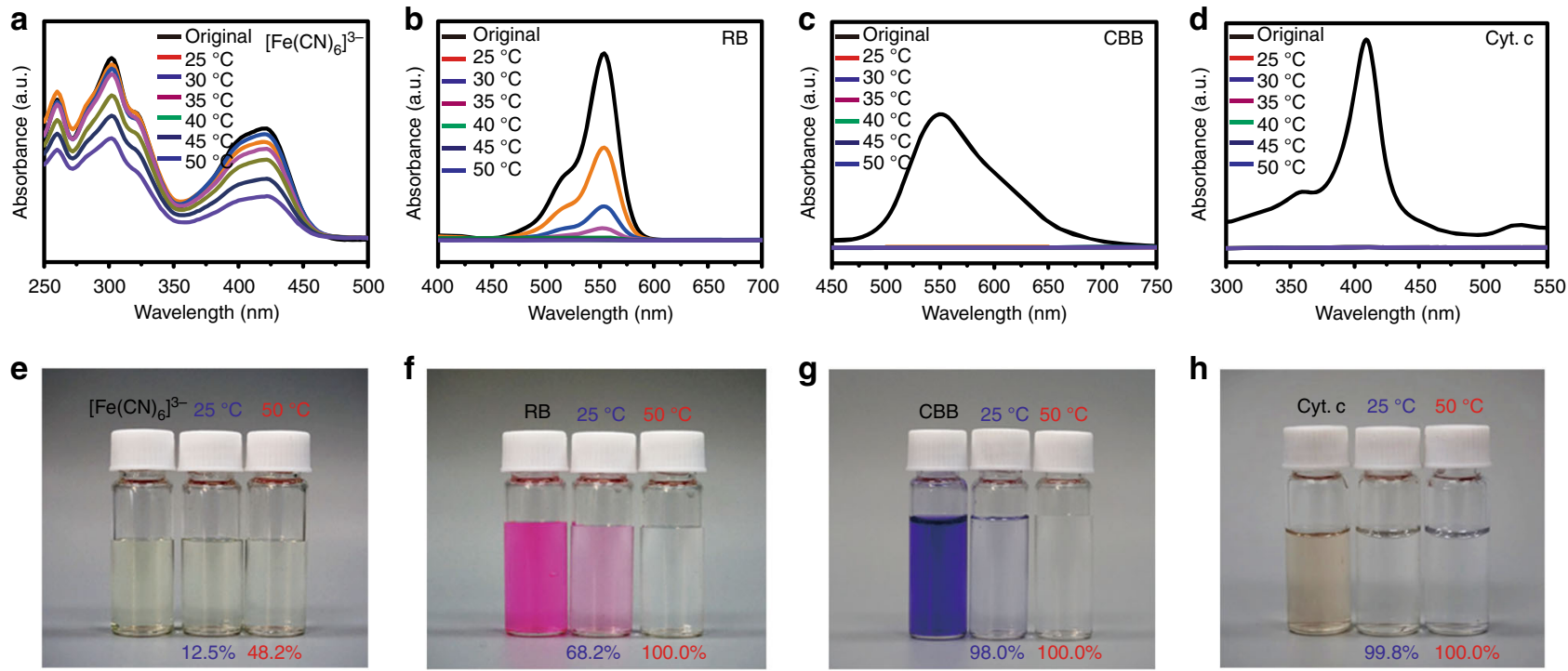

Fig. 5 P-GOMs for single molecule separation. a-d The UV-vis absorption spectra before and after filtering $\left[\mathrm{Fe}(\mathrm{CN})_{6}\right]^{3-}$ aqueous solutions, rhodamine $\mathrm{B}$ (RB) aqueous solutions, coomassie brilliant blue (CBB) aqueous solutions, and cytochrome $\mathrm{c}$ (Cyt. C) aqueous solutions from $25^{\circ} \mathrm{C}$ to $50^{\circ} \mathrm{C}$. e-h The digital photos of original solutions and the filtrate obtained at $25^{\circ} \mathrm{C}$ and $50{ }^{\circ} \mathrm{C}$ of $\left[\mathrm{Fe}(\mathrm{CN})_{6}\right]^{3-}, \mathrm{RB}, \mathrm{CBB}$, and $\mathrm{Cyt}$. C aqueous solutions

(1b), and $-154.6 \mathrm{~kJ} \mathrm{~mol}^{-1}(2 \mathrm{~b})$. For the consecutive reactions, three and four NIPAM monomers react with one or two functionalized graphene sheets. In this situation, all the reactions are exothermic. However, the reaction enthalpies for the reactions involving one functionalized graphene sheet were -158.3 (3a) and $-262.7 \mathrm{~kJ} \mathrm{~mol}^{-1}(4 \mathrm{a})$, whereas the reactions involving two graphene sheets were much more exothermic with reaction enthalpies of -254.5 (3b) and $-396.0 \mathrm{~kJ} \mathrm{~mol}^{-1}$ (4b). The higher exothermicities of the later reactions could be partly attributed to the stronger van der Waals interactions between the graphene sheets and the side chain of the PNIPAM. These computational results indicate that the polymerization reaction between graphene and PNIPAM will prefer to be terminated by another functionalized graphene sheet as case (1) showed in Fig. 4a (Supplementary Note 3). We have shown that this is the case for 1-4 NIPAM molecules, however this trend is expected to continue for larger numbers of NIPAM molecules.

Separation performance of the P-GOMs. To validate the results of temperature tunable channel size of P-GOMs discussed above, we designed a separation experiment to test the lamellar space change at different temperatures by using small molecules with different sizes. Here we employed five ions/molecules, $\mathrm{Cu}^{2+}(0.8 \mathrm{~nm}),\left[\mathrm{Fe}(\mathrm{CN})_{6}\right]^{3-}(0.9 \times 0.9 \mathrm{~nm})$, rhodamine $\mathrm{B}(\mathrm{RB}$, $1.8 \times 1.4 \mathrm{~nm})$, coomassie brilliant blue $(\mathrm{CBB}, 2.7 \times 1.8 \mathrm{~nm})$, and cytochrome c (Cyt. c, $2.5 \times 2.5 \times 3.7 \mathrm{~nm})^{22,29,57}$. The rejection rates of the five ions/molecules through the P-GOMs from $25^{\circ} \mathrm{C}$ to $50^{\circ} \mathrm{C}$ were measured. The zeta potential results (Supplementary Fig. 17) demonstrate that the P-GOMs have few anionic sites for adsorption of the positively charged molecules and rejection of the negatively charged molecules because of the relatively low absolute value of the zeta potential. Thus size-dependent rejection rates nearly indicate the nanochannels size change of P-GOMs ${ }^{58,59}$. The results in Figs. 5 and 6 a demonstrate that the nanochannel sizes of the P-GOMs gradually decrease with the increasing temperature (Supplementary Table 2). These results not only verified the results of quantum chemical calculations, but also demonstrated that the negative temperature-response P-GOMs could be promising for precise molecular separation (Supplementary Figs. 18 and 19, Supplementary Note 4).
Moreover, we suppose that the P-GOMs could realize gradient separation of multiple molecules with different sizes.

The multiple molecules separation schematic diagram is illustrated in Fig. 6b. The smallest molecule $\mathrm{A}$ is separated out at $50{ }^{\circ} \mathrm{C}$, leaving the medium and largest molecules (B and $\mathrm{C}$ ) in the retentate. Then the temperature changes to $25^{\circ} \mathrm{C}$ to separate the medium size molecule from the large molecule. Thus, we can separate multiple molecules with different sizes using the P-GOMs by simple regulating the temperature. Subsequently, we designed a separation scenario using the mixed molecules solution, containing $\mathrm{Cu}^{2+}, \mathrm{RB}$, and Cyt. c (Fig. 6c, d). Figure 6e shows the UV-vis absorption spectra of filtrates obtained at $50{ }^{\circ} \mathrm{C}$ and $25^{\circ} \mathrm{C}$ and retentate. For the $\mathrm{Cu}^{2+}$, it only existed in the filtrate obtained at $50{ }^{\circ} \mathrm{C}$ according to the results of inductively coupled plasma optical emission spectrometry. In the filtrate obtained at $50{ }^{\circ} \mathrm{C}$, there were trace $\mathrm{RB}$ and Cyt. $\mathrm{c}$ according to its UV-vis absorption spectrum. For RB, it enriched in the filtrate obtained at $25^{\circ} \mathrm{C}$. Therefore, only the largest Cyt. c remained in the retentate obtained at $25^{\circ} \mathrm{C}$. The results verified the selective separation performance of the P-GOMs to the mixed ions/ molecules solution (Supplementary Fig. 20).

We have reported a smart temperature-response gating membrane composed of GO and PNIPAM for hydraulic permeability applications. The membranes have negative response coefficients with a gating ratio of $\sim 7$. What's more, the gating membranes could realize gradual small/medium/large molecule separation by simple step-wise tuning of the temperature. This study provides insight toward the design of smart gating membranes, showing promise for applications in fluid transport systems, microfluidic chip systems, and molecular separation devices.

\section{Methods}

Materials. GO was prepared by a modified Hummer's method. Ethanol, 2,2'Azobis(2-methylpropionitrile) ( $\geq 99.0 \%$, recrystallization), $N, N$-dimethylformamide (DMF, $\geq 99.8 \%$, anhydrous), copper (II) chloride $\left(\mathrm{CuCl}_{2}\right)$, potassium ferricyanide $\left(\mathrm{K}_{3}\left[\mathrm{Fe}(\mathrm{CN})_{6}\right]\right)$, rhodamine $\mathrm{B}(\mathrm{RB})$, coomassie brilliant blue, cytochrome $\mathrm{c}$ (Cyt. c), and raffinose were all purchased from Aladdin Chemistry Co., Ltd. (Shanghai, China) and used as received. Maltopentaose and maltoheptaose (HPLC) were provided by Miragen (USA). NIPAM was provided by Sigma-Aldrich (Shanghai, China) and purified by recrystallization from $n$-hexane ( $\geq 99 \%$, Aladdin). PNIPAM $\left(M_{n} \sim 20,000-40,000\right)$ was provided by Sigma-Aldrich. 


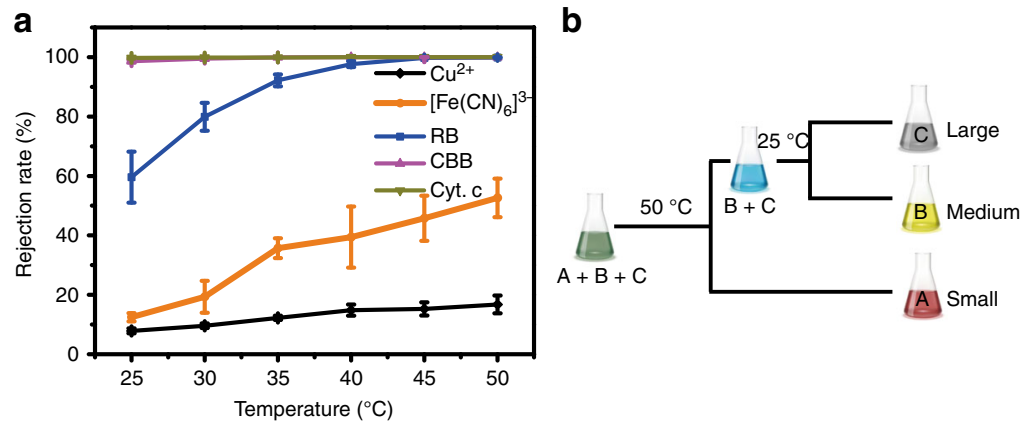

C
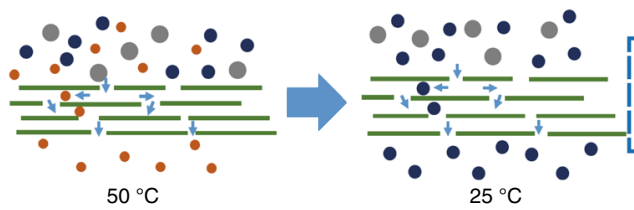

$\mathrm{Cu}^{2+}(0.8 \mathrm{~nm})$ - $\mathrm{RB}(1.8 \times 1.4 \mathrm{~nm})$ Cyt. c $(2.5 \times 2.5 \times 3.7 \mathrm{~nm})$

d

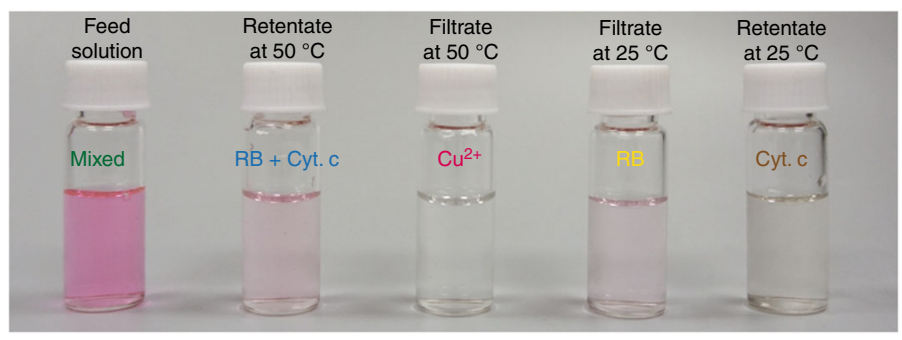

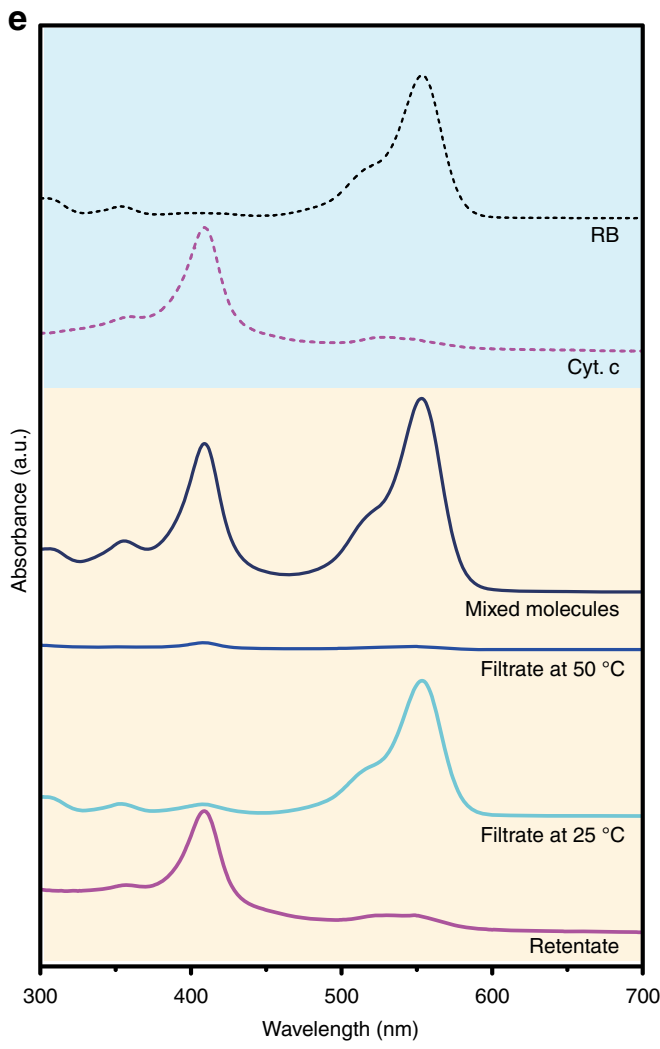

Fig. 6 P-GOMs for multiple molecules separation. a Temperature-dependent rejection rates of P-GOMs to $\mathrm{Cu}^{2+}$, $\left[\mathrm{Fe}(\mathrm{CN})_{6}\right]^{3-}, \mathrm{RB}, \mathrm{CBB}$, and $\mathrm{Cyt}$. C, implying smaller channel size of the P-GOMs with the increasing temperature. Error bars, s.d. $(n=3)$. $\mathbf{b}$ Schematic diagram of the separation mechanism of P-GOMs towards mixed molecules solution. c Separation of mixed molecules solution, containing $\mathrm{Cu}^{2+}, \mathrm{RB}$, and Cyt. c. $\mathbf{d}$ The digital photos of the mixed molecules feed solution, retentate at $50{ }^{\circ} \mathrm{C}$, filtrate at $50{ }^{\circ} \mathrm{C}$, filtrate at $25^{\circ} \mathrm{C}$, and retentate at $25^{\circ} \mathrm{C}$. e The UV-vis absorption spectra of pure RB aqueous solution, Cyt. c aqueous solution, mixed molecules solution, filtrate obtained at $50^{\circ} \mathrm{C}$ and $25^{\circ} \mathrm{C}$, and retentate at $25^{\circ} \mathrm{C}$

Preparation of P-GO dispersion. The P-GO were synthesized by free-radical polymerization. Typically, GO $(0.05 \mathrm{~g})$ was first fully dispersed in DMF $(50 \mathrm{ml})$ by stirring for $1 \mathrm{~h}$ and ultrasonic treatment for $2 \mathrm{~h}$. Afterwards, NIPAM $(50 \mathrm{mmol}$, $5.659 \mathrm{~g})$ and 2,2'-Azobis (2-methylpropionitrile) $(0.056 \mathrm{~g})$ were added to the dispersion, respectively. The mixture was subsequently transferred to a $100 \mathrm{ml}$ Schlenk flask followed by stirring for 30 mins. Next, the reagent mixture was subjected to three freeze-pump-thaw cycles and was then placed in a $65^{\circ} \mathrm{C}$ oil bath. The reaction was terminated after $12 \mathrm{~h}$ by immersing the flask into liquid nitrogen. The obtained solution was diluted with $250 \mathrm{ml}$ DI water and transferred into centrifuge tubes followed by $1 \mathrm{~h}$ centrifugation at the speed of $10,000 \mathrm{r} \mathrm{min}^{-1}$ $(10,744 \mathrm{~g})$. The precipitates located in the bottom of centrifuge tubes after centrifugation were collected and washed three times each with $300 \mathrm{ml}$ DI water and $300 \mathrm{ml}$ ethanol. To make sure NIPAM monomer and the PNIPAM not bound to $\mathrm{GO}$ were completely separated from the P-GO, the precipitates were purified through dialysis in DI water for 3 days using dialysis bag (MW cutoff: 30,000 ). Then the P-GO were obtained after freezing dry of the dispersion for 2 days with a freezing dryer. A brownish transparent, stable P-GO dispersion $\left(0.1 \mathrm{mg} \mathrm{ml}^{-1}\right)$ was obtained by adding $0.01 \mathrm{~g}$ of the as-prepared P-GO in $100 \mathrm{ml}$ DI water and stirring for $2 \mathrm{~h}$. Note, no ultrasound treatment was needed to obtain the well-dispersed P-GO aqueous suspension during the whole synthesis process except dispersing GO into DMF at first.

Preparation of GO dispersion. GO $(0.009 \mathrm{~g})$ was added into $100 \mathrm{ml}$ DI water followed by stirring for $1 \mathrm{~h}$ and ultrasonic treatment for $2 \mathrm{~h}$ to obtain a welldispersed $\mathrm{GO}$ aqueous dispersion $\left(0.09 \mathrm{mg} \mathrm{ml}^{-1}\right)$.

Preparation of GO blending with PNIPAM dispersion. PNIPAM (0.021 g) was added into $100 \mathrm{ml} \mathrm{GO}$ aqueous dispersion $\left(0.09 \mathrm{mg} \mathrm{ml}^{-1}\right)$ and stirred for $6 \mathrm{~h}$ to obtain the homogeneous GO blending with PNIPAM dispersion.

Fabrication of P-GOMs, GOMs, and GO blending with PNIPAM membranes. For the preparation of a P-GOM, $6 \mathrm{ml}$ (except discussing as variable) P-GO aqueous dispersion $\left(0.1 \mathrm{mg} \mathrm{m}^{-1}\right)$ was filtered through a CA membrane by pressuredriven filtration (Millipore 8010, Millipore, USA) with a filter area of $2.83 \mathrm{~cm}^{2}$ under the pressure of $100 \mathrm{kPa}$. Once no P-GO dispersion left above the formed membrane, $10 \mathrm{ml}$ DI water was immediately added to the filtration cell to keep the
P-GOM in a wet state. The mass of GO in a P-GOM was $\sim 0.00018 \mathrm{~g}$ based on the calculation from the TGA and differential thermogravimetrie curves as described in Supplementary Note 1 . Thus, the procedure was basically the same as the P-GOM for the fabrication of GOM or GO blending with PNIPAM membrane, except using $2 \mathrm{ml} \mathrm{GO}$ dispersion $\left(0.09 \mathrm{mg} \mathrm{m}^{-1}\right)$ or $2 \mathrm{ml} \mathrm{GO}$ blending with PNIPAM dispersion (containing $0.00018 \mathrm{~g} \mathrm{GO}$ ) instead of the P-GO dispersion. The GO mass in three kinds of membranes were all fixed at $\sim 0.18 \mathrm{mg}$. The average values were obtained by measuring three independent samples for each. What is more, in order to keep the GOM stable in water, a negative pressure was applied to the pressure-driven filtration when added $10 \mathrm{ml}$ DI water above the GOM until putting pressure on the membrane.

\section{Permeability performances of P-GOMs, GOMs and GO blending with PNI-}

PAM membranes. For the temperature-responsive hydraulic permeability experiments, a water bath was used to regulate the temperature of the membrane The filtration device contained the formed membrane and $10 \mathrm{ml}$ DI water was put into the water bath with desired temperature. After standing for 30 mins, $100 \mathrm{kPa}$ pressure generated by $\mathrm{N}_{2}$ was applied to the membrane. The weight of water penetrated through the membrane was recorded every $30 \mathrm{mins}$, and the water permeance was calculated using the value which kept constant for $>90$ mins.

For the photo-responsive hydraulic permeability experiments, a vacuum filtration system combined with an $808 \mathrm{~nm}$ NIR laser was used.

Molecular separation experiments of P-GOMs. While for the molecule separation experiments, the aqueous solutions of $100 \mathrm{mgl}^{-1} \mathrm{CuCl}_{2}, 40 \mathrm{mgl}^{-1}$ $\mathrm{K}_{3}\left[\mathrm{Fe}(\mathrm{CN})_{6}\right], 4 \mathrm{mgl}^{-1} \mathrm{RB}$ (Supplementary Fig. 21), $40 \mathrm{mgl}^{-1}$ coomassie brilliant blue (Supplementary Fig. 22), $100 \mathrm{mgl}^{-1}$ Cyt. c, $200 \mathrm{mgl}^{-1}$ raffinose, $200 \mathrm{mgl}^{-1}$ maltopentaose and $200 \mathrm{mgl}^{-1}$ maltoheptaose were used instead of DI water, and the water bath contained the filtration device with a stir bar was placed on a magnetic heated stirrer in case of concentration polarization. After certain time, the filtrates were collected. The rejection rate $(R)$ was calculated using the following equation: 
where $c_{\mathrm{p}}$ and $c_{\mathrm{o}}$ are the concentrations of the molecule in the permeates and original solutions.

For the mixed molecules separation experiments, $5 \mathrm{ml}$ aqueous solution contained $100 \mathrm{mgl}^{-1} \mathrm{Cu}^{2+}, 4 \mathrm{mgl}^{-1} \mathrm{RB}$, and $100 \mathrm{mgl}^{-1} \mathrm{Cyt}$. $\mathrm{c}$ was used as the feed solution. First, the filtration device was put in the $50^{\circ} \mathrm{C}$ water bath, after standing for 30 mins, $100 \mathrm{kPa}$ pressure was applied to the membrane. After collecting $\sim 4.5$ $\mathrm{ml}$ filtrate, $4.5 \mathrm{ml}$ DI water was added into the filtration device, followed by the second separation at $50^{\circ} \mathrm{C}$. The temperature was altered to

$25^{\circ} \mathrm{C}$ until no $\mathrm{Cu}^{2+}$ could be detected in the filtrate at $50^{\circ} \mathrm{C}$. Like the separation process at $50^{\circ} \mathrm{C}$, after several separations at $25^{\circ} \mathrm{C}$, the filtrate contained only RB could be obtained. The retentate contained only Cyt. c could be obtained as well in the end.

Computational details. To simplify the calculations, we used a methyl radical $\left(\mathrm{CH}_{3}{ }^{\bullet}\right)$ to mimic the experimentally carbon related radical (namely $\mathrm{CN}\left(\mathrm{CH}_{3}\right)_{2} \mathrm{C} \bullet$ ) as the initiator. A graphene sheet containing 36 carbon atoms, $\mathrm{C}_{36} \mathrm{H}_{16}$, was used to study the polymerization reaction instead of GO used in experiment because the polymer chains were grafted to graphitic surface utilizing the double bonds of surfaces in the free-radical polymerization ${ }^{42}$. Using graphene sheet did not influence the results, however, substantially saved computational cost. The initiation, propagation, and termination steps of a free-radical polymerization reaction were carried out by using NIPAM as a monomer and $\mathrm{CH}_{3}$ as the initiator. The reaction of a methyl radical with graphene and the subsequent reaction of the newly formed graphene-methyl radical with NIPAM were examined for the initiation step. The subsequent reaction of graphene-methyl- NIPAM radical with another NIPAM monomer was examined for the propagation step. The termination step was examined by considering the reaction of the resultant radical with another methyl functionalized graphene sheet. For every step, geometries of the reactants and products were optimized using density functional theory method with a hybrid functional B3LYP $60-62$ and a 6-31 G(d, p) basis set. Empirical D3 dispersion corrections are included using the Becke-Johnson damping potential as recommended in Ref.63 (denoted by the suffix-D3). Bulk solvent effects of DMF ( $\varepsilon=$ 37.2) have been included using charge-density based SMD continuum solvation model at the same level of theory ${ }^{64}$. All calculations were performed by using Gaussian 09 package ${ }^{65}$.

Characterization. Digital photographs were shot by a digital camera. Scanning electron microscope images were obtained on a scanning electron microscopy (JSM-7500F, JEOL, Japan) equipped with a energy dispersive spectroscopy detector. AFM images were carried out on a Bruker Dimension Icon (Japan). Diluted GO or P-GO aqueous dispersion was spin-coated (2000 rpm) on freshly cleaved mica and dried at room temperature to make the AFM samples. Thermographic images were obtained with a FLIR TG165 imaging IR thermometer (FLIR Systems, Inc., OR, USA). The values of contact angle were measured on an OCA20 machine (Dataphysics, Germany) at room temperature. ATR-FTIR spectra were measured by an infrared microspectrometer (Thermo Scientific Nicolet iN10, USA). XPS characterizations were performed by ESCALAB 250Xi (Thermo Fisher Scientific, USA) equipped with a monochromatic Al Ka X-ray source. Raman spectroscopy measurements were performed using a Lab-RAM HR800 (Horiba JobinYvon, France) with an incident laser of $514 \mathrm{~nm}$ wavelength. The XRD analyses were carried out by an X-ray diffractometer (XRD-6000, Shimadzu, Japan), using $\mathrm{Cu} \mathrm{Ko}$ radiation $(\lambda=0.15418 \mathrm{~nm})$. The ${ }^{1} \mathrm{H}$ nuclear magnetic resonance spectra were obtained on a Bruker Avance III $700 \mathrm{MHz}$ NMR spectrometer (Bruker Biospin, Rheinstetten, Germany). TGA was performed on a thermogravimetric analyzer (STA449F3, Netzsch, Germany), with a temperature increase rate of $10 \mathrm{~K} \mathrm{~min}^{-1}$ under nitrogen from $25^{\circ} \mathrm{C}$ to $750{ }^{\circ} \mathrm{C}$. The thicknesses of P-GOMs were measured by Bruker DektakXT Stylus Profiler (Germany). The concentrations of $\mathrm{Cu}^{2+}$ in the solution were analyzed using inductively coupled plasma optical emission spectrometry (ICP-OES, Optima 5300DV, PerkinElmer, USA). The concentrations of raffinose, maltopentaose and maltoheptaose in the solution were analyzed by ion chromatography equipped with a CarboPac PA200 $3 \times 250$ column. UV-vis absorption spectra were obtained with a Shimadzu UV-3600 spectrometer (Japan) equipped with a thermal controller. Zeta potentials were analyzed by DelsaNano C Zeta Potential Analyzer (Beckman Coulter Inc., USA). Tensile mechanical properties were measured at room temperature $\left(25^{\circ} \mathrm{C}\right)$ via a Shimadzu AGS-X Tester (Japan) with a $20 \mathrm{~N}$ load cell at a loading rate of $1 \mathrm{~mm} \mathrm{~min}^{-1}$. The samples were cut into strips with a width of $3 \mathrm{~mm}$ and length of $10 \mathrm{~mm}$, and the thicknesses of all samples were calculated by scanning electron microscope thickness measuring instrument.

Data availability. All data generated or analyzed during this study are present in the main text and the Supplementary Information. Additional data are available from the authors on reasonable request.

Received: 27 June 2017 Accepted: 13 November 2017

Published online: 08 December 2017

\section{References}

1. Collatz, G. J., Ball, J. T., Grivet, C. \& Berry, J. A. Physiological and environmental regulation of stomatal conductance, photosynthesis and transpiration: a model that includes a laminar boundary layer. Agric. For. Meteorol. 54, 107-136 (1991).

2. Park, C. H. et al. Nanocrack-regulated self-humidifying membranes. Nature 532, 480-483 (2016).

3. Chu, L. Y., Li, Y., Zhu, J. H. \& Chen, W. M. Negatively thermoresponsive membranes with functional gates driven by zipper-type hydrogen-bonding interactions. Angew. Chem. Int. Ed. 44, 2124-2127 (2005).

4. Liu, Z., Wang, W., Xie, R., Ju, X. J. \& Chu, L. Y. Stimuli-responsive smart gating membranes. Chem. Soc. Rev. 45, 460-475 (2016).

5. Schepelina, O. \& Zharov, I. PNIPAAM-modified nanoporous colloidal films with positive and negative temperature gating. Langmuir 23, 12704-12709 (2007).

6. Yameen, B. et al. Ionic transport through single solid-state nanopores controlled with thermally nanoactuated macromolecular gates. Small $\mathbf{5}$, 1287-1291 (2009).

7. Guo, W. et al. Current rectification in temperature-responsive single nanopores Chem. Phys. Chem. 11, 859-864 (2010).

8. Morones-Ramírez, J. R. Bioinspired synthesis of optically and thermally responsive nanoporous membranes. NPG Asia Mater. 5, e52 (2013).

9. Hou, X. Smart gating multi-scale pore/channel-based membranes. Adv. Mater. 28, 7049-7064 (2016)

10. Halperin, A., Kröger, M. \& Winnik, F. M. Poly (N-isopropylacrylamide) phase diagrams: fifty years of research. Angew. Chem. Int. Ed. 54, 15342-15367 (2015).

11. Wang, N., Zhao, Y. \& Jiang, L. Low-cost, thermoresponsive wettability of surfaces: poly (n-isopropylacrylamide)/polystyrene composite films prepared by electrospinning. Macromol. Rapid Commun. 29, 485-489 (2008).

12. Junk, M. J. et al. EPR spectroscopic characterization of local nanoscopic heterogeneities during the thermal collapse of thermoresponsive dendronized polymers. Angew. Chem. Int. Ed. 49, 5683-5687 (2010).

13. Li, H. et al. Ultrathin, molecular-sieving graphene oxide membranes for selective hydrogen separation. Science 342, 95-98 (2013).

14. Kurapati, R. \& Raichur, A. M. Near-infrared light-responsive graphene oxide composite multilayer capsules: a novel route for remote controlled drug delivery. Chem. Commun. 49, 734-736 (2013).

15. Sun, P., Wang, K. \& Zhu, H. Recent developments in graphene-based membranes: structure, mass-transport mechanism and potential applications. Adv. Mater. 28, 2287 (2016).

16. Zhao, J. et al. Fabricating graphene oxide-based ultrathin hybrid membrane for pervaporation dehydration via layer-by-layer self-assembly driven by multiple interactions. J. Membr. Sci. 487, 162-172 (2015).

17. Koenig, S. P., Wang, L., Pellegrino, J. \& Bunch, J. S. Selective molecular sieving through porous graphene. Nat. Nanotechnol. 7, 728-732 (2012).

18. Kim, H. W. et al. Selective gas transport through few-layered graphene and graphene oxide membranes. Science 342, 91-95 (2013).

19. Joshi, R. et al. Precise and ultrafast molecular sieving through graphene oxide membranes. Science 343, 752-754 (2014)

20. Huang, L., Li, Y., Zhou, Q., Yuan, W. \& Shi, G. Graphene oxide membranes with tunable semipermeability in organic solvents. Adv. Mater. 27, 3797-3802 (2015).

21. Huang, L. et al. Reduced graphene oxide membranes for ultrafast organic solvent nanofiltration. Adv. Mater. 28, 8669-8674 (2016).

22. Gao, S. J., Qin, H., Liu, P. \& Jin, J. SWCNT-intercalated GO ultrathin films for ultrafast separation of molecules. J. Mater. Chem. A 3, 6649-6654 (2015).

23. Liu, N. et al. Ultralight free-standing reduced graphene oxide membranes for oil-in-water emulsion separation. J. Mater. Chem. A 3, 20113-20117 (2015).

24. Mi, B. Graphene oxide membranes for ionic and molecular sieving. Science 343 740-742 (2014).

25. Abraham, J. et al. Tunable sieving of ions using graphene oxide membranes. Nat. Nanotechnol. 12, 546-550 (2017).

26. Han, Y., Xu, Z. \& Gao, C. Ultrathin graphene nanofiltration membrane for water purification. Adv. Funct. Mater. 23, 3693-3700 (2013).

27. Long, Y. et al. Molecule channels directed by cation-decorated graphene oxide nanosheets and their application as membrane reactors. Adv. Mater. 29, 1606093 (2017).

28. Fathizadeh, M., Xu, W. L., Zhou, F., Yoon, Y. \& Yu, M. Graphene oxide: a novel 2-dimensional material in membrane separation for water purification. $A d v$ Mater. Interfaces 4, 1600918 (2017).

29. Huang, H. et al. Ultrafast viscous water flow through nanostrand-channelled graphene oxide membranes. Nat. Commun. 4, 2979 (2013).

30. Abozar, A. et al. Large-area graphene-based nanofiltration membranes by shear alignment of discotic nematic liquid crystals of graphene oxide. Nat. Commun. 7, 10891 (2016). 
31. Hou, X., Hu, Y., Grinthal, A., Khan, M. \& Aizenberg, J. Liquid-based gating mechanism with tunable multiphase selectivity and antifouling behaviour. Nature 519, 70-73 (2015).

32. Hu, L. et al. Photothermal-responsive single-walled carbon nanotube-based ultrathin membranes for on/off switchable separation of oil-in-water nanoemulsions. ACS Nano 9, 4835-4842 (2015).

33. Huang, $\mathrm{H}$. et al. Salt concentration, $\mathrm{pH}$ and pressure controlled separation of small molecules through lamellar graphene oxide membranes. Chem. Commun. 49, 5963-5965 (2013).

34. Liu, R. et al. Graphene oxide membrane for liquid phase organic molecular separation. Carbon N. Y. 77, 933-938 (2014).

35. Sun, P. et al. Electro-and magneto-modulated ion transport through graphene oxide membranes. Sci. Rep. 4, 6798 (2014).

36. Wang, Y. et al. Graphene-directed supramolecular assembly of multifunctional polymer hydrogel membranes. Adv. Funct. Mater. 25, 126-133 (2015).

37. Dong, J., Weng, J. \& Dai, L. The effect of graphene on the lower critical solution temperature of poly (N-isopropylacrylamide). Carbon N. Y. 52, 326-336 (2013).

38. Hung, W. S. et al. Cross-linking with diamine monomers to prepare composite graphene oxide-framework membranes with varying d-spacing. Chem. Mater. 26, 2983-2990 (2014).

39. Moon, I. K., Lee, J., Ruoff, R. S. \& Lee, H. Reduced graphene oxide by chemical graphitization. Nat. Commun. 1, 73 (2010).

40. Kundu, A., Nandi, S., Das, P. \& Nandi, A. K. Fluorescent graphene oxide via polymer grafting: an efficient nanocarrier for both hydrophilic and hydrophobic drugs. ACS Appl. Mat. Interfaces 7, 3512-3523 (2015).

41. Qi, J., Lv, W., Zhang, G., Zhang, F. \& Fan, X. Poly (N-isopropylacrylamide) on two-dimensional graphene oxide surfaces. Polym. Chem. 3, 621-624 (2012).

42. Kan, L., Xu, Z. \& Gao, C. General avenue to individually dispersed graphene oxide-based two-dimensional molecular brushes by free radical polymerization. Macromolecules 44, 444-452 (2010).

43. Liu, J. et al. A graphene-like oxygenated carbon nitride material for improved cycle-life lithium/sulfur batteries. Nano. Lett. 15, 5137-5142 (2015).

44. Kim, Y. S. et al. Thermoresponsive actuation enabled by permittivity switching in an electrostatically anisotropic hydrogel. Nat. Mater. 14, 1002-1007 (2015).

45. Pan, Y., Bao, H., Sahoo, N. G., Wu, T. \& Li, L. Water-soluble poly (Nisopropylacrylamide) -graphene sheets synthesized via click chemistry for drug delivery. Adv. Funct. Mater. 21, 2754-2763 (2011).

46. Bergman, T. L., Incropera, F. P., DeWitt, D. P. \& Lavine, A. S. Fundamentals of heat and mass transfer (Hobeken, NJ, USA: Wiley, 2011).

47. Wei, N., Peng, X. \& Xu, Z. Breakdown of fast water transport in graphene oxides. Phys. Rev. E Stat. Nonlin. Soft Matter Phys. 89, 012113 (2014).

48. Nair, R., Wu, H., Jayaram, P., Grigorieva, I. \& Geim, A. Unimpeded permeation of water through helium-leak-tight graphene-based membranes. Science 335, 442-444 (2012).

49. Acik, M. et al. Unusual infrared-absorption mechanism in thermally reduced graphene oxide. Nat. Mater. 9, 840-845 (2010).

50. Zhong, Y. et al. Elimination of photon quenching by a transition layer to fabricate a quenching-shield sandwich structure for $800 \mathrm{~nm}$ excited upconversion luminescence of $\mathrm{Nd}^{3+}$-sensitized nanoparticles. Adv. Mater. 26, 2831-2837 (2014)

51. Zong, L., Li, M. \& Li, C. Bioinspired coupling of inorganic layered nanomaterials with marine polysaccharides for efficient aqueous exfoliation and smart actuating hybrids. Adv. Mater. 29, 1604691 (2017).

52 . Lv, Ja et al. Photocontrol of fluid slugs in liquid crystal polymer microactuators. Nature 537, 179-184 (2016)

53. Chu, L. Y., Niitsuma, T. \& Yamaguchi, T. \& Nakao, S. I. Thermoresponsive transport through porous membranes with grafted PNIPAM gates. AlChE J. 49, 896-909 (2003)

54. Li, Y. et al. Thermoresponsive gating characteristics of poly (Nisopropylacrylamide)-grafted porous poly (vinylidene fluoride) membranes. Ind. Eng. Chem. Res. 43, 2643-2649 (2004).

55. Li, W. et al. Low toxic, thermoresponsive dendrimers based on oligoethylene glycols with sharp and fully reversible phase transitions. Chem. Commun. 0, 5948-5950 (2008).

56. Mylvaganam, K. \& Zhang, L. In situ polymerization on graphene surfaces. J. Phys. Chem. C. 117, 2817-2823 (2013).

57. Tansel, B. Significance of thermodynamic and physical characteristics on permeation of ions during membrane separation: Hydrated radius, hydration free energy and viscous effects. Sep. Purif. Technol. 86, 119-126 (2012).
58. Patil, S., Sandberg, A., Heckert, E., Self, W. \& Seal, S. Protein adsorption and cellular uptake of cerium oxide nanoparticles as a function of zeta potential. Biomaterials 28, 4600-4607 (2007).

59. Zhu, S. et al. Strongly green-photoluminescent graphene quantum dots for bioimaging applications. Chem. Commun. 47, 6858-6860 (2011).

60. Lee, C., Yang, W. \& Parr, R. G. Development of the Colle-Salvetti correlationenergy formula into a functional of the electron density. Phys. Rev. B 37, 785 (1988).

61. Becke, A. D. Density-functional thermochemistry. III. The role of exact exchange. J. Chem. Phys. 98, 5648-5652 (1993).

62. Stephens, P., Devlin, F., Chabalowski, C. \& Frisch, M. J. Ab initio calculation of vibrational absorption and circular dichroism spectra using density functional force fields. J. Phys. Chem. 98, 11623-11627 (1994).

63. Grimme, S., Ehrlich, S. \& Goerigk, L. Effect of the damping function in dispersion corrected density functional theory. J. Comput. Chem. 32, 1456-1465 (2011).

64. Marenich, A. V., Cramer, C. J. \& Truhlar, D. G. Universal solvation model based on solute electron density and on a continuum model of the solvent defined by the bulk dielectric constant and atomic surface tensions. J. Phys. Chem. B 113, 6378-6396 (2009).

65. Frisch, M. et al. Gaussian 09, Revision D. 01; (Gaussian: Wallingford, CT, USA, 2009).

\section{Acknowledgements}

We are grateful for the financial support from the National Natural Science Foundation of China $(21774005,21433012,21374001,21222309)$, the National Youth Talent Support Program, the Program for New Century Excellent Talents in University of China, the Fundamental Research Funds for the Central Universities, the China Scholarship Council (201506025110) and the National Instrumentation Program (2013YQ120355). The work at Harvard was supported by the NSF (DMR-1310266) and the Harvard MRSEC (DMR 1420570).

\section{Author contributions}

Y.Z. conceived the study and designed the experiments. J.C.L. performed most of the experiments. L.J.Y and A.K. performed the density functional theory calculations. W.L., W.X.Z., and Q.F.C. provided some valuable scientific suggestions about the temperatureresponsive property of the P-GOMs. F.Y.G., and L.L.H. performed the tensile experiments and analyzed the data. J.C.L. and N.W. wrote the text. Y.Z., N.W., L.J., and D.A.W revised the paper.

\section{Additional information}

Supplementary Information accompanies this paper at https://doi.org/10.1038/s41467017-02198-5.

Competing interests: The authors declare no competing financial interests.

Reprints and permission information is available online at http://npg.nature.com/ reprintsandpermissions/

Publisher's note: Springer Nature remains neutral with regard to jurisdictional claims in published maps and institutional affiliations.

Open Access This article is licensed under a Creative Commons Attribution 4.0 International License, which permits use, sharing, adaptation, distribution and reproduction in any medium or format, as long as you give appropriate credit to the original author(s) and the source, provide a link to the Creative Commons license, and indicate if changes were made. The images or other third party material in this article are included in the article's Creative Commonslicense, unless indicated otherwise in a credit line to the material. If material is not included in the article'sCreative Commons license and your intended use is not permitted by statutory regulation or exceeds the permitted use, you will need to obtain permission directly from the copyright holder. To view a copy of this license, visit http://creativecommons.org/ licenses/by/4.0/.

(C) The Author(s) 2017 